\title{
A Note on Punctuation
}

In what follows, double quotation marks indicate citations of other authors, terms used in a commonly accepted sense to which I am referring as quotations (though no particular source may be provided), or, in some few cases, phrases cited from earlier parts of my book. Single marks, except when they indicate a quotation within a quotation, are used for other emphases-most of ten either to indicate the inappropriateness of some habitually used term or to signal that a term taken from one discursive logic (or class of discourse, as it will be called hereafter) is being unavoidably but unsuitably applied to a different such logic. 

The Discourse of Modernism 
